\title{
VERZEICHNIS DER MITARBEITER DIESES BANDES
}

Prof. Dr. Diana Behler, Department of Germanics DH-30, University of Washington, Seattle, Washington 98195 , U.S.A.

Christina Berti, Via Rosmini, 44-46, I-55049 Viareggio (LU)

Dr. Marco Brusotti, Selchower Str. 7, W-1000 Berlin 44

Prof. Dr. Adrian Del Caro, Germanic and Slavic Languages \& Literatures, Campus Box 276, University of Colorado at Boulder, Boulder, CO 80309-0276, U.S.A.

Dr. Paolo D’Iorio, Via Emilia, 80, I-55046 Querceta (Lucca)

Dr. Domenico M. Fazio, Università di Lecce, Istituto di Filosofia, via V. M. Stampacchia, I-73100 Lecce

Dr. Stephen Houlgate, Department of Philosophy, DePaul University, Chicago, Illinois, U.S.A.

Dr. Henry Kerger, Dodostr. 21, W-4400 Münster

Lic. Hans Erich Lampl, Neubergq. 20, N-0367 Oslo

Andrea Orsucci, Scuola Normale Superiore, Piazza dei Cavalieri, I-56100 Pisa

Florian Roth, M.A., Auerstr. 34, W-8000 München 5

Dr. Michael Platt, Friends of the Republic, Sugar Hill, East Wallingford, Vermont 05742, U.S.A.

Dr. Richard Schottky, Am Heckendorn 103, W-5600 Wuppertal 2

lic. phil. Martin Stingelin, Deutsches Seminar, Universität Basel, Engelhof, Nadelberg 4, $\mathrm{CH}-4051$ Basel

Prof. Dr. Hubert Treiber, M.A., Lic., Fachbereich Rechtswissenschaft, Universität Hannover, Hanomagstr. 8, W-3000 Hannover 91

Prof. Dr. Aldo Venturelli, Via Asiago, 6, I-60124 Ancona 
